\title{
Effect of Feldenkrais exercises on dual task postural control in older adults
}

This article was published in the following Dove Press journal:

Clinical Interventions in Aging

4 July 2014

Number of times this article has been viewed

\author{
Gerhild Ullmann' \\ Harriet G Williams ${ }^{2}$ \\ 'Social and Behavioral Sciences, \\ University of Memphis, School \\ of Public Health, Memphis, TN, USA; \\ ${ }^{2}$ Exercise Science, University of South \\ Carolina, Columbia, SC, USA
}

\section{Dear editor}

Agmon et $\mathrm{al}^{1}$ recently published an interesting systematic review of interventions to improve dual-task postural control in older adults. Given that many everyday activities (eg, walking and carrying groceries) require dual-task postural control, this is an important topic. This type of research is integral to expanding scientific knowledge in the field of interventions. The authors describe the methods of the review process clearly. However, in our opinion, adherence to the stated methods is not always evident.

First, in the abstract, the authors claim to select randomized and nonrandomized controlled studies. In contrast with the stated study selection, Table 1 shows that not all of the 22 studies included are in compliance with these design requirements; several uncontrolled studies were examined, including an earlier uncontrolled study by the authors.

Second, at least one randomized controlled trial that fulfills the selection and inclusion criteria has not been considered in the review. Our randomized controlled trial examined the effects of a Feldenkrais intervention on balance, gait, and single-task and dual-task mobility. ${ }^{2}$ This study can be found in electronic databases with several of the mentioned search terms. Our research met all inclusion and selection criteria: participants were healthy, community-dwelling older adults aged 65 years and older; the intervention was conducted in a clinical setting; the one-hour Feldenkrais sessions were held three times per week for 5 weeks; one of the outcome measures was mobility under dual-task conditions (in our Feldenkrais study we used the Timed Up and Go with an added cognitive [TUGc] ${ }^{3}$ task, and several studies included in Agmon's review used the same TUGc measure for examining dual-task performance, see Table 4 of the systematic review ${ }^{1}$ ); the article was published in English; and the randomized controlled trial excluded participants with neurologic disorders.

After completion of the Feldenkrais intervention, participants improved significantly on balance $(P=0.030)$, mobility under single-task conditions $(P=0.042)$, and showed positive changes in mobility under dual-task conditions $(P=0.067){ }^{2}$

The authors of the review underline in their discussion the prominence of dualtask training for improving dual-task performance. However, an important outcome of our publication is that a broad spectrum of activities that are part of the Feldenkrais method can result in improvements in both single-task performances and dual-task performance. From our perspective, this is an important piece of the puzzle and should be noted if we want to design evidence-based interventions based on current scientific knowledge.
Correspondence: Gerhild Ullmann Social and Behavioral Sciences, University of Memphis, School of Public Health, 3825 DeSoto Avenue, Robison Hall, Memphis, TN 38I52, USA

$\mathrm{Tel}+|90| 678$ I340

Fax+l901678 1715

Email ullmann I@memphis.edu 


\section{Disclosure}

The authors report no conflicts of interest in this communication.

\section{References}

1. Agmon M, Belza B, Nguyen HQ, Logsdon R, Kelly VE. A systematic review of interventions conducted in clinical or community settings to improve dual-task postural control in older adults. Clin Interv Aging. 2014;9:477-492.
2. Ullmann G, Williams HG, Hussey J, Durstine JL, McClenaghan BA. Effects of Feldenkrais exercises on balance, mobility, balance confidence, and gait performance in community-dwelling adults age 65 and older. J Altern Complement Med. 2010;16:97-105.

3. Shumway-Cook A, Brauer S, Woollacott M. Predicting the probability for falls in community-dwelling older adults using the Timed Up and Go Test. Phys Ther. 2000;80:896-903. 


\section{Author's reply}

Maayan Agmon'

Basia Belza ${ }^{2}$

Huong Q Nguyen ${ }^{2,3}$

Rebecca G Logsdon ${ }^{2}$

Valerie E Kelly ${ }^{4}$

'The Cheryl Spencer Department of Nursing, Faculty of Social Welfare and Health Studies, University of Haifa, Israel; ${ }^{2} \mathrm{School}$ of Nursing, University of Washington, Seattle, WA, ${ }^{3}$ Department of Research and Evaluation, Kaiser Permanente, CA, ${ }^{4}$ School of Medicine, University of Washington, Seattle, WA, USA

Correspondence: Maayan Agmon

University of Haifa, Abba Hushi I, Haifa, Israel

Tel +97254900 I609

$\mathrm{Fax}+972548288017$

Email agmon.mn@gmail.com

\section{Dear editor}

We thank Dr Ullmann and colleagues for their comments and the opportunity to clarify a number of points from our recent review of interventions to improve dual-task postural control in older adults. As they note, it is critical to expand our understanding of evidence-based interventions in this area, given the functional relevance of dual-task postural control in daily life.

Our systematic review aimed to examine the effectiveness of interventions on dual-task postural control among healthy older adults and to identify key elements of effective training protocols. Dr Ullmann and colleagues note the clear description of our methods. However, they express concern regarding adherence to these methods because this search did not identify their randomized controlled trial examining the effects of Feldenkrais exercises on balance, mobility, and balance confidence in older adults.

To address this, we reviewed our search methods to identify possible reasons for why this paper was not identified. It appears that the primary reason was due to inclusion of the search term "dual-task". As stated in our manuscript, our search terms were grouped into four categories of terms related to: dual-task, older adults, intervention, and gait or balance. These four categories were used for all searches, so the term "dual-task" was a consistent part of every search. Although our search methodology did not identify the paper in question, on further review we were able to identify this manuscript by removing the term "dual-task" from our search. In addition to this keyword search, we identified one additional paper through a manual search of the references from included studies. Thus, we also examined whether the study in question might have been overlooked in the references of included papers. However, it does not appear that this manuscript was cited by any of our included studies or related systematic reviews in this field. Given the sparse research in this area, we chose to include two uncontrolled trials, as noted in this commentary; however, a manuscript that otherwise met our inclusion criteria was not identified with our search. This highlights the critical roles that keywords for a specific study and initial search terms in a systematic review play in identification of articles of interest.

Of concern, then, is whether the work by Ullmann and colleagues impacts the overall conclusions of our systematic review. Their study incorporated several outcome measures, including but not limited to those related to balance (tandem stance) and mobility (Timed Up and Go without a concurrent task [TUG] and with a cognitive task [TUGc]), to determine the effects of Feldenkrais exercise compared with a wait list control in older adults. A significant interaction between group (experimental, control) and time (pre-test, post-test) was observed for tandem stance $(P=0.030)$, indicating balance improvements in the experimental group but not in the control group. A significant group $\times$ time interaction was also observed for the TUG $(P=0.042)$, demonstrating maintenance of mobility in the experimental group compared with declines in the control group. There was a nonsignificant group $\times$ time interaction $(P=0.067)$ for TUGc; however, the authors completed a post hoc analysis that demonstrated an improvement in dual-task mobility for the experimental group but no significant change for the control group. Thus, this study demonstrates benefits for single-task balance and mobility and a trend toward improved dual-task mobility in the experimental group compared with the control group.

We do not feel that these findings change the overall conclusions of our review. In our manuscript, we noted that the ability to synthesize data across studies was limited based on heterogeneity of participant characteristics, study designs, and outcome measures. That challenge remains when considering the work by Ullman and colleagues. For example, because cognitive task performance was not reported, it is unclear whether benefits in dual-task mobility came at the expense of declines in dual-task cognitive performance. We feel that the significant effects of this intervention on singletask postural control and a trend toward effects on dual-task postural control demonstrated in the work by Ullman and colleagues do not change our overall conclusion that dualtask postural control appears to be optimally modified by specific dual-task training.

Ultimately, though, we feel that this area will best be moved forward by continued clinical research that 
incorporates more high-quality randomized controlled trials that align with CONSORT (Consolidated Standards of Reporting Trials) guidelines (http://www.consortstatement.org/), including the recommendations for rehabilitation research, as well as agreement regarding a uniform set of outcome measures, including agreed upon dual-task protocols that allow for comparison across and synthesis of data from multiple trials. We appreciate the opportunity to consider this additional study and eagerly anticipate additional research that may shed light on this critical area.

\section{Disclosure}

The authors report no conflicts of interest in this work.

\section{Publish your work in this journal}

Clinical Interventions in Aging is an international, peer-reviewed journal focusing on evidence-based reports on the value or lack thereof of treatments intended to prevent or delay the onset of maladaptive correlates of aging in human beings. This journal is indexed on PubMed Central, MedLine,
CAS, Scopus and the Elsevier Bibliographic databases. The manuscript management system is completely online and includes a very quick and fair peer-review system, which is all easy to use. Visit http://www.dovepress. com/testimonials.php to read real quotes from published authors. 